\section{Singapore synchrotron completes its first year of routine operation}

The Singapore Synchrotron Light Source (SSLS) building project was approved in 1997. In 1999 the building was completed and the Helios 2 storage ring moved into place, and in 2000 the accelerator system was commissioned and beamline construction began. In October 2001, user pilot operation started with a phase-contrast imaging beamline (PCI). A micro/nanofabrication facility (LiMiNT), a soft X-ray facility for surface, interface and nanoscience (SINS), and the X-ray development and demonstration beamline (XDD) were added. Routine user operation was achieved by 2003. A beamline for infrared spectro/microscopy (ISMI) will be ready soon.

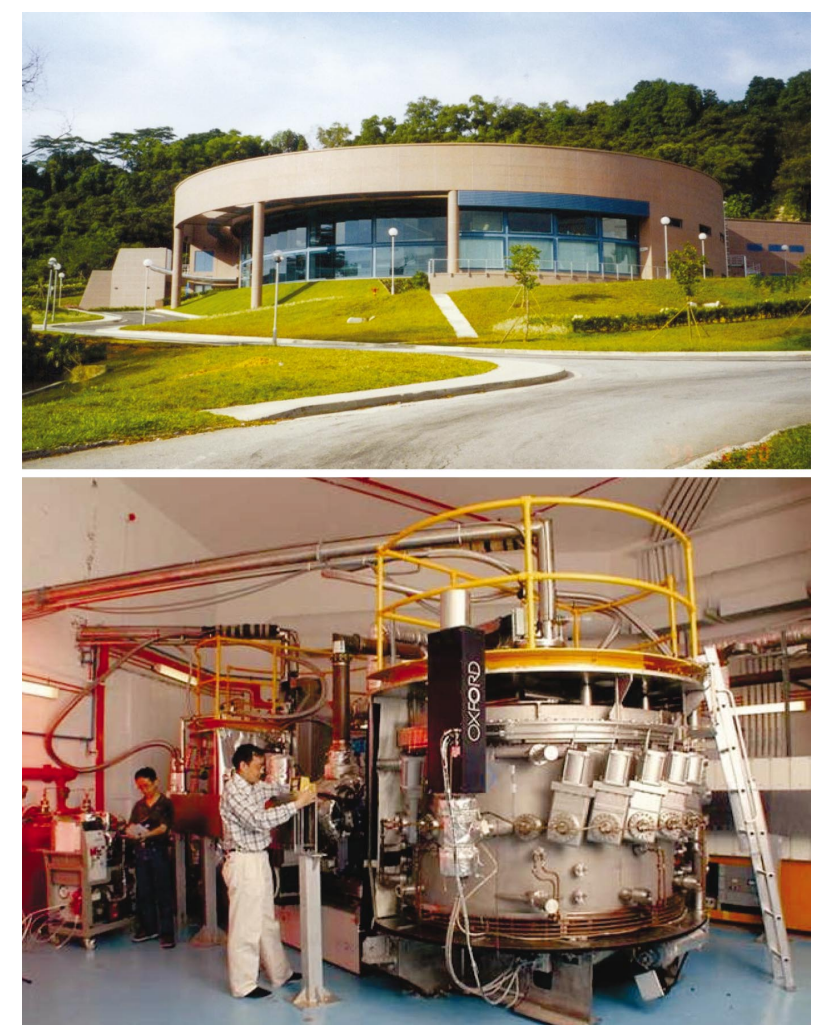

Top: SSLS building on the campus of the National University of Singapore. Bottom: Helios 2 storage ring inside the shielding vault.

SSLS is developing the general-purpose synchrotron light source capability of the compact superconducting storage ring Helios 2, which was manufactured by Oxford Instruments in close collaboration with Daresbury Laboratory in the early 1990s for the computerchip-making industry. $700 \mathrm{MeV}$ electrons orbiting through $4.5 \mathrm{~T}$ dipoles produce a very useful spectrum from about $10 \mathrm{keV}$ to the far infrared. Several beamlines have come into routine operation and include the SINS (surface, nanostructures and interface science, see photograph top right) and the XDD (X-ray development and demonstration) beamlines. XDD has been used for example in demonstrating the high quality of $K$-edge XAFS of a thin $\mathrm{Cu}$ plate on $\mathrm{Si}$. With photoemission spectroscopy, X-ray magnetic circular dichroism and X-ray absorption fine-structure spectroscopy from $50 \mathrm{eV}$ to $1.2 \mathrm{keV}$, SINS is extensively used for thin-film and nanostructures characterization for such fields as magnetic data storage and self-assembly-based nanomanufacturing. Forthcoming in situ scanning tunneling and atomic force microscopy (STM/AFM) will enhance its competitive edge. Like all national synchrotron radiation facilities the SSLS user community has grown, from a few to almost 100 in a brief period.

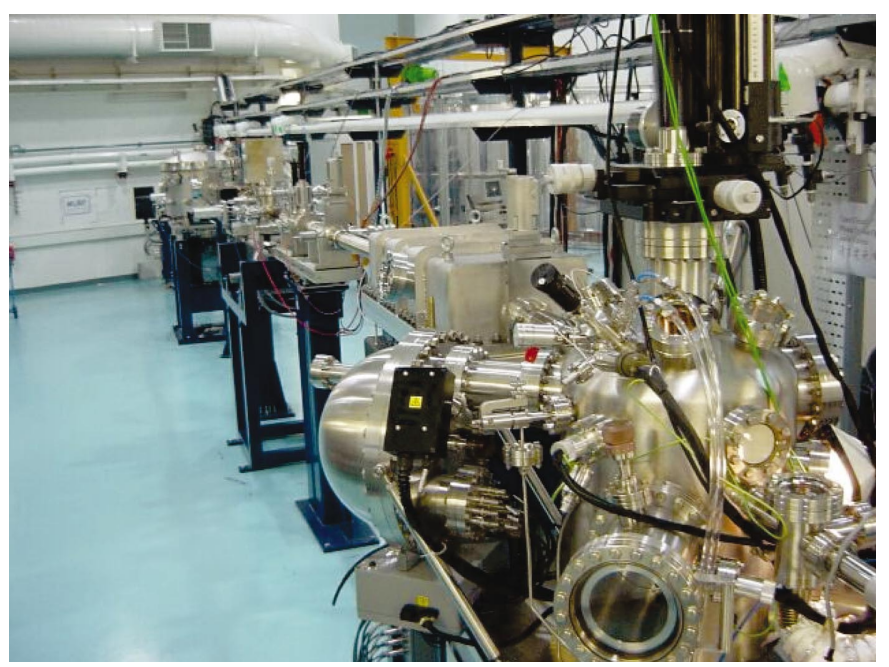

SINS beamline at SSLS

\section{SINO-EU workshop on metalloproteins gives rise to metal- logenomics}

An intensive workshop was held at the Chinese Academy of Sciences' Institute of High Energy Physics on 27-28 June 2004. The workshop focused on the advances in synchrotron radiation studies of metalloproteins. It brought together experts of two powerful synchrotron radiation structural methods (XAFS and protein crystallography) and metalloproteins from pathogens. The combined approach to metalloproteins, making $>30 \%$ of the genome, has been pioneered by the EU groups coming together through a series of workshops over the last five years (e.g. see the dedicated special issue of the Journal of Synchrotron Radiation, January 2003), which has now attracted the attention of leading groups in the USA and more recently from China. Proteins from Mycobacterium tuberculosis (MTb), already the focus of several programmes in the EU, attracted special attention. It was felt that this combined integrated approach is required to study this class of proteins in such an important pathogen of healthcare importance, and an initiative on metallogenomics with this unique science focus was urgently needed. The workshop was organised by Ziyu Wu and Isabella Ascone from the Beijing Synchrotron Facility and Orsay. The meeting was opened by Professor Chen, Director of the Chinese Acadamey of Sciences' Institute of High Energy Physics.

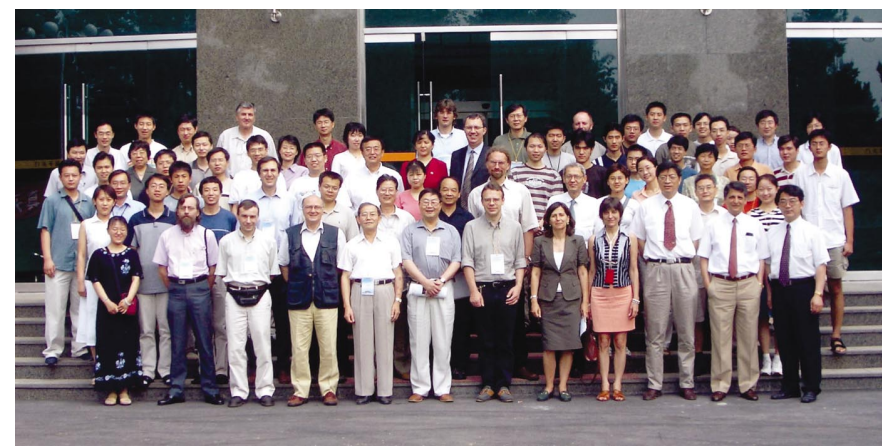

The delegates of the workshop. On the extreme right is Professor Ziyu Wu and third from the right is Professor Chen. 